\title{
Simulation of diffusio-phoretic motion of colloidal particle suppressed by bound solutes within adsorption shell
}

\section{Jiachen Wei}

To cite this article: Jiachen Wei (2020): Simulation of diffusio-phoretic motion of colloidal particle suppressed by bound solutes within adsorption shell, Molecular Physics, DOI: 10.1080/00268976.2020.1718226

To link to this article: https://doi.org/10.1080/00268976.2020.1718226

View supplementary material $\asymp$

曲 Published online: 26 Jan 2020.

Submit your article to this journal $₫$

ЏIll Article views: 3

Q View related articles $₫$

View Crossmark data $\circlearrowright$ 


\title{
Simulation of diffusio-phoretic motion of colloidal particle suppressed by bound solutes within adsorption shell
}

\author{
Jiachen Wei ${ }^{a, b}$

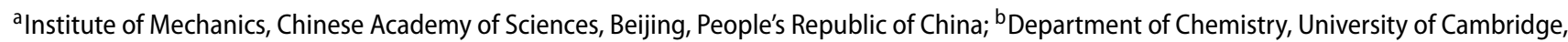 \\ Cambridge, UK
}

\begin{abstract}
Colloidal particle submerged in a non-equilibrium fluid with a concentration gradient of solutes experiences diffusio-phoresis. Such directional transport originates from the driving forces that exert on the fluid in a microscopic boundary layer surrounding the colloid. Based on a simple model of spherical colloid fixed in a concentration gradient of solutes, molecular dynamics simulations are performed to determine the interaction parameters that maximise the diffusio-phoretic mobility, which cannot be properly measured by conventional continuum theory. The diffusio-phoretic mobility is found to depend non-monotonically on the strength of the interaction between the colloid and solutes, due to the presence of bound solutes within adsorption shell that cannot contribute to diffusio-phoresis. The results also show that the phoretic mobility depends sensitively on the density of solutes in bulk, due to the uneven distribution of excess particles surrounding the colloid at a microscopic level. The simulations suggest that diffusio-phoresis may in principle be applied to the selective transport, separation and purification for colloidal systems. By substituting the spherical colloid with other realistic macromolecules, the model could provide results that are quantitatively comparable with experiments.
\end{abstract}

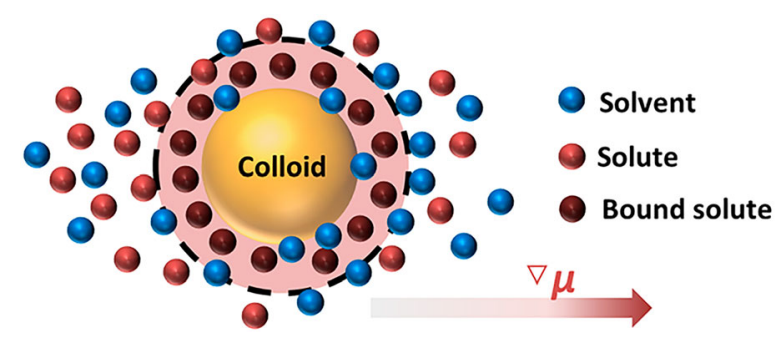

ARTICLE HISTORY

Received 6 November 2019 Accepted 9 January 2020

\section{KEYWORDS}

Diffusio-phoresis; molecular dynamics; colloid; phoretic transport

\section{Introduction}

The phoretic effects are a class of transport phenomena induced by gradients of chemical potential [1-8], temperature [9-22], charge [23-28], etc. Diffusio-phoresis describes the spontaneous migration in a gradient of solute concentration [29-32], which plays important roles in separation science [33-37], pattern formation $[5,32,38,39]$ and even dynamics in a living cell [40].

The migration of colloidal particles by phoresis has long been described by continuum models and hydrodynamics $[2,8,25,30,41,42]$, which provide an adequate explanation for the experimental results. For diffusio-phoresis with short-ranged interactions, however, the Stokesian hydrodynamics no longer holds near the fluid-colloid interface, as it fails to picture the effects of density and enthalpy fluctuations that emerge at microscopic level $[43,44]$. Inspired by the previous works on diffusio- and thermo-osmosis $[45,46]$, in this article molecular dynamics simulations are implemented to describe the local structure and dynamics of the fluid. The gradient of solute concentration can be generated either explicitly with fixed source and sink of solute particles $[14,44,47,48]$, or implicitly by applying external forces on each particle $[45,46]$.

CONTACT Jiachen Wei weijiachen@Inm.imech.ac.cn Institute of Mechanics, Chinese Academy of Sciences, Beijing 100190, People's Republic of China; Department of Chemistry, University of Cambridge, Cambridge CB21EW, UK.

(-) Supplemental data for this article can be accessed here. https://doi.org/10.1080/00268976.2020.1718226 
Based on these techniques of non-equilibrium molecular simulations, results for the diffusio-phoretic motion of a colloidal particle in a very weak gradient of solute concentration are reported in the remainder of this article. The dependence of the phoretic mobility on the strength and range of the solute-colloid interactions, solute density and the size of the colloidal particle is presented, which can hardly be quantitatively determined with mesoscopic models. Note that the long-range columbic interactions are not taken into account, so the simulated system is to measure the diffusio-phoresis of a colloidal particle in the gradient of neutral solutes. The manuscript is organised as follows: the next section introduces the model and simulation methods. Simulation results and discussions are then presented. The last section is the summary.

\section{Simulation method}

\subsection{Model}

Molecular dynamics (MD) is implemented to simulate the diffusio-phoresis. All MD simulations are carried out in LAMMPS [49]. The fluid particle $i$ of type $\alpha$ (solvent) or $\beta$ (solute) interacts with another fluid particle $j$ through the Lennard-Jones (LJ) 12-6 potential that is truncated and shifted at $r_{i j}^{t}=4.0$ :

$$
\begin{aligned}
U_{i j}^{L J}\left(r_{i j}\right) & =4 \epsilon_{i j}\left[\left(\frac{\sigma_{i j}}{r_{i j}}\right)^{12}-\left(\frac{\sigma_{i j}}{r_{i j}}\right)^{6}\right], \\
U_{i j}\left(r_{i j}\right) & = \begin{cases}U_{i j}^{L J}\left(r_{i j}\right)-U_{i j}^{L J}\left(r_{i j}^{t}\right) & \text { for } r_{i j}<r_{i j}^{t}, \\
0 & \text { for } r_{i j} \geq r_{i j}^{t},\end{cases}
\end{aligned}
$$

with $\epsilon_{i j}$ and $\sigma_{i j}$ the LJ constants. Here and in what follows, I use reduced units, based on $\epsilon_{\alpha \alpha}, \sigma_{\alpha \alpha}$ and $m_{\alpha}$ (mass of the solvent particle).

Morse potential is used to model the interactions between the colloid $c$ and fluid particle $i$ of type $\alpha$ or $\beta$ :

$$
\begin{aligned}
U_{i c}^{M}\left(r_{i c}\right) & =\epsilon_{i c}\left[e^{-2\left(r_{i c}-\sigma_{i c}\right) / \kappa_{i c}}-2 e^{-\left(r_{i c}-\sigma_{i c}\right) / \kappa_{i c}}\right], \\
U_{i c}\left(r_{i c}\right) & = \begin{cases}U_{i c}^{M}\left(r_{i c}\right)-U_{i c}^{M}\left(r_{i c}^{t}\right) & \text { for } r_{i c}<r_{i c}^{t}, \\
0 & \text { for } r_{i c} \geq r_{i c}^{t},\end{cases}
\end{aligned}
$$

where $\epsilon_{i c}, \kappa_{i c}$, and $\sigma_{i c}$, respectively, control the interaction strength, interaction range, and the position of the minimum. Note that the subscript $c$ denotes the fixed colloid, and the potential is truncated and shifted at $r_{i c}^{t}=7.0$. See Table 1 for the full list of interaction parameters used in this paper (unless otherwise specified).

The concentration gradient can be explicitly simulated by a source and sink region with the fixed number density of solute [48] in a $50.00 \times 19.73 \times h$ box with periodic
Table 1. Table of parameters for interactions between different particles (unless specified).

\begin{tabular}{lcccc}
\hline Lennard-Jones $i-j$ & $\epsilon_{i j}$ & $\sigma_{i j}$ & - & $r_{i j}^{t}$ \\
\hline solvent-solvent $\alpha-\alpha$ & 1.0 & 1.0 & - & 4.0 \\
solvent-solute $\alpha-\beta$ & 1.0 & 1.0 & - & 4.0 \\
solute-solute $\beta-\beta$ & 1.0 & 1.0 & - & 4.0 \\
Morse $i$-colloid & $\epsilon_{i c}$ & $\sigma_{i c}$ & $\kappa_{i c}$ & $r_{i c}^{t}$ \\
solvent-colloid $\alpha-c$ & 1.0 & 3.0 & 0.5 & 7.0 \\
solute-colloid $\beta-c$ & $\epsilon_{\beta c}$ & 3.0 & 0.5 & 7.0
\end{tabular}

boundary conditions, as shown in Figure 1(a). The box height $h$ in the $z$-direction is varied to keep the pressure at constant. The flow rate of the fluid is measured, whilst keeping the colloid fixed at the origin.

As shown in Figure 1(b), the concentration gradient can also be represented implicitly $[45,48]$ by applying (different) gradient forces $f_{i}$ on the solvent and solute particles:

$$
f_{i}=\left(\frac{-\partial \mu_{i}^{\text {bulk }}}{\partial \rho_{i}}\right)_{P} \cdot \nabla \rho_{i},
$$

where $\mu_{i}^{\text {bulk }}$ is the chemical potential and $\rho_{i}$ the number density. The system dimensions are $19.73 \times 19.73 \times$ $h$, and the colloid is fixed at the origin, providing the counteracting force $F_{c}=-\left(f_{\alpha} N_{\alpha}+f_{\beta} N_{\beta}\right)$ to ensure that there is no net force on the fluid. In the remainder of this paper, model with implicit gradient is used unless otherwise specified (due to its better accuracy and efficiency).

\subsection{Simulation details}

All simulations are performed in NPT ensemble, with fixed pressure at $P=0.012$, and temperature at $T=0.845$. The number of particles is fixed at $N=12308$ and $N=7124$ for the system with explicit and implicit gradient, respectively. Nosé-Hoover thermostat with damping constant for temperature $100 d t$ and Nosé-Hoover barostat with damping constant for pressure $1000 d t$ are implemented, with $d t=0.001$ representing the length of the time step. As it may be useful to compare simulations with experimental results, in Table 2 quantities in reduced units used in this work are converted into real units. For all real units, the mass and LJ parameters of solvent particles are chosen as those of argon atom.

All initial configurations are prepared as follows. First, $N-1$ solvent particles are uniformly distributed on FCC crystal lattice at low densities to prevent overlap. $N_{\beta}=$ $\rho_{\beta} V$ random solvent particles are then replaced with solute particles, where $V$ is the volume of the simulation box and $\rho_{\beta}$ is the density of solute. The system is then compressed in $z$-direction and quenched to the desired pressure. At $\epsilon_{\beta c}=2.6$, The typical height of the 

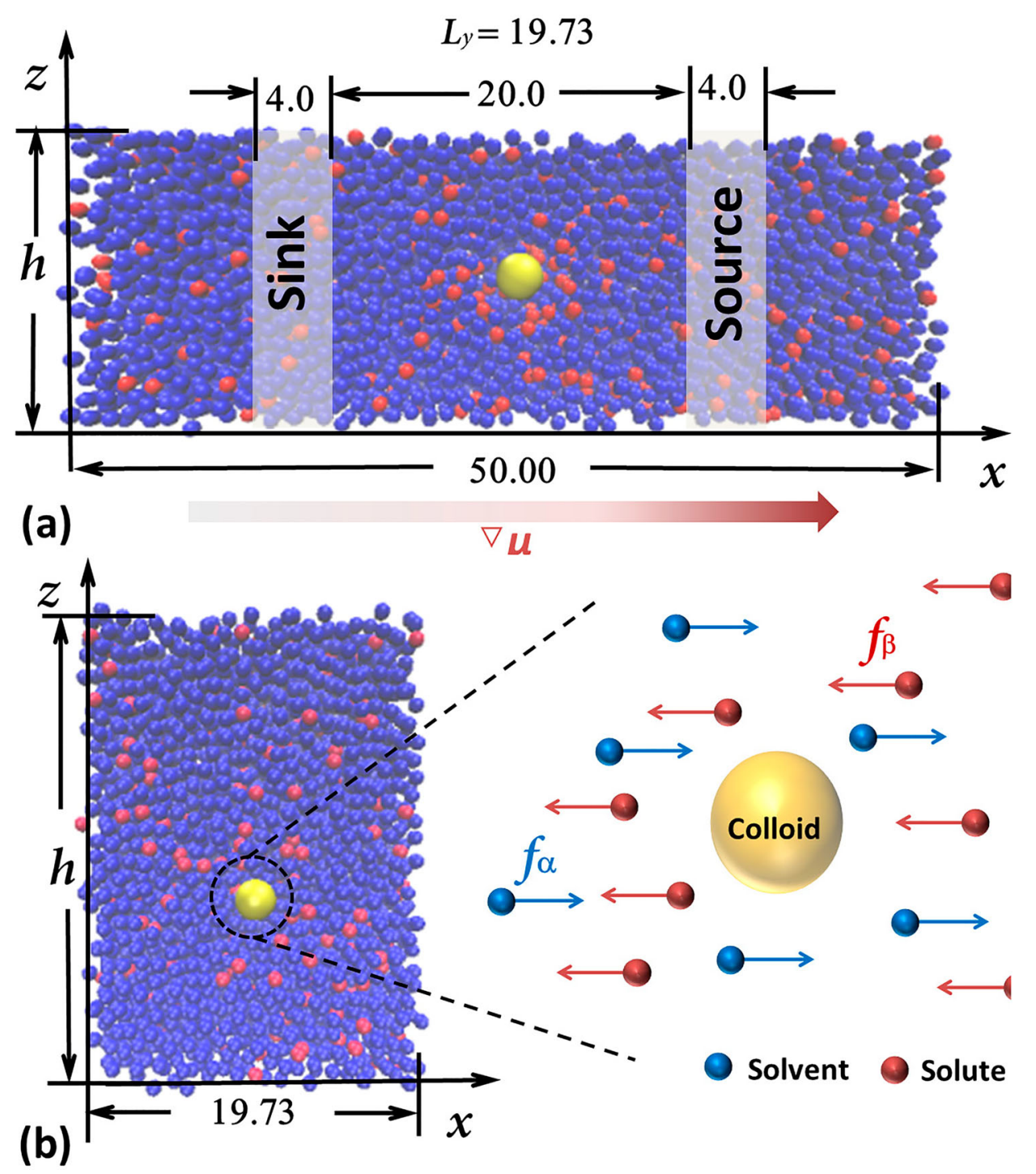

Figure 1. Slice snapshot of the model systems with (a) explicit or (b) implicit concentration gradient of solutes.

Table 2. Comparison of quantities measured in reduced and real units (in terms of argon atom).

\begin{tabular}{lcc}
\hline Quantities & In reduced unit & In real unit \\
\hline$\sigma_{\alpha \alpha}$ & 1.0 & $0.341 \mathrm{~nm}$ \\
$\epsilon_{\alpha \alpha}$ & 1.0 & $10.30 \mathrm{meV}$ \\
$m_{\alpha}$ & 1.0 & $39.95 \mathrm{~g} / \mathrm{mol}$ \\
$d t$ & 0.001 & $2.164 \mathrm{fs}$ \\
$P$ & 0.012 & $0.5 \mathrm{MPa}$ \\
$T$ & 0.845 & $101 \mathrm{~K}$ \\
\hline
\end{tabular}

box is $h=23.92$ for simulations with implicit gradient, and $h=16.34$ for explicit gradient.

For simulations with explicit concentration gradient, the identities of the fluid particles within the source $\left(\rho_{\beta}=0.07\right)$ or sink $\left(\rho_{\beta}=0.03\right)$ regions are reset every $5 \times 10^{2}$ simulation steps to maintain a constant gradient and $\rho_{\beta} \sim 0.05$ at $x=0$. For simulations with an implicit concentration gradient, the identities of solvent and solute particles in the bulk (i.e. far away from the colloidal particle) are allowed to interchange every 500 simulation steps to keep the density of solute in bulk $\rho_{\beta}^{b u l k}$ constant for at least $4 \times 10^{7}$ simulation steps. Gradient forces $f_{i}$ along the $x$-direction are then applied to the solvent and solute particles. Subsequently, a long run (at least $2 \times 10^{8}$ steps) is performed to obtain the flow rate, density profile, etc., which are collected by averaging over $10^{5}$ output configurations separated by $10^{3}$ simulation steps. For both cases, a solute concentration gradient of $\left|\nabla \rho_{\beta}\right|=0.002$ is generated.

Based on the Green-Kubo formula, I determine the radial auto-correlation for the shear terms of stress tensor, $\xi(r)$, which is written as

$$
\begin{aligned}
\xi(r)= & \frac{V}{k_{B} T} \int_{0}^{\infty}\left[\left\langle p_{x y}(0, r) p_{x y}(t, r)\right\rangle\right. \\
& \left.+\left\langle p_{x z}(0, r) p_{x z}(t, r)\right\rangle+\left\langle p_{y z}(0, r) p_{y z}(t, r)\right\rangle\right] / 3 \mathrm{~d} t,
\end{aligned}
$$




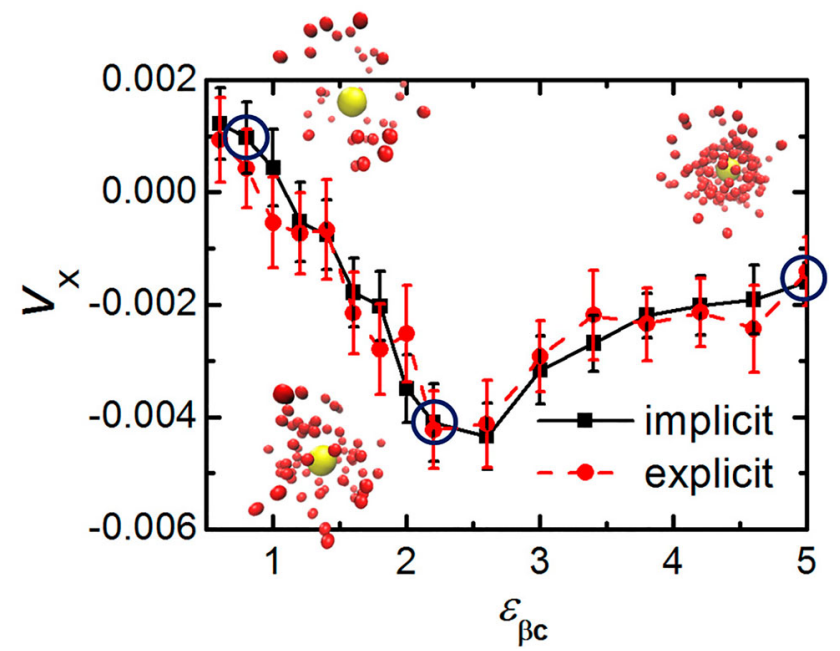

Figure 2. The dependence of velocity of fluids in $x$-direction, $v_{x}$, on the interaction strength between solute and colloid, $\epsilon_{\beta c}$, obtained from simulations with implicit and explicit gradient. The density of solute in bulk is fixed at $\rho_{\beta}^{\text {bulk }}=0.05$. Typical simulation snapshots of solutes around the colloid at $\epsilon_{\beta c}=0.8, \epsilon_{\beta c}=2.2$ and $\epsilon_{\beta c}=5.0$ are presented.

where $p_{x y}(t, r), p_{x z}(t, r)$ and $p_{y z}(t, r)$ are off-diagonal stress tensors averaged over particles at a distance $r$ to the fixed colloid, and $k_{B}$ is the Boltzmann constant.

\section{Result and discussion}

\subsection{Interaction}

Figure 2 shows that for all values of $\epsilon_{\beta c}$, the interaction strength between solute and colloid, $v_{x}$, the velocity in the direction of phoretic motion obtained from simulations with imposed gradient forces agrees well with that obtained using an explicit concentration source and sink. It is found that $v_{x}$ depends non-monotonically on $\epsilon_{\beta c}$. For $\epsilon_{\beta c}<2.5$, the magnitude of $v_{x}$ increases with $\epsilon_{\beta c}$. This is because the number of adsorbed solute particles, and hence total gradient force exerted on them, increases with $\epsilon_{\beta c}$. If the density profile around the colloid is radially symmetric, the net force density equals $F_{x}(r)=$ $\rho_{\alpha}(r) f_{\alpha}-\rho_{\beta}(r) f_{\beta}$ and $f_{\alpha} / f_{\beta}=\rho_{\beta}^{b u l k} / \rho_{\alpha}^{b u l k}$. In that case, an excess of solute $(\beta)$ would imply $F_{x}(r)<0$ and an excess of solvent, $F_{x}(r)>0$. The maximum velocity $\left(v_{x}^{m}=-0.004\right)$ is obtained for $\epsilon_{\beta c} \approx 2.5$. When the solute-colloid interaction is further increased, the magnitude of $v_{x}$ decreases and reaches $v_{x} \sim-0.002$ at $\epsilon_{\beta c}=$ 5.0. This is because a sticky shell of solute particles is effectively trapped around the colloid for larger values of $\epsilon_{\beta c}$, which does not contribute to diffusio-phoresis. The formation of the shell-region is demonstrated by the typical simulation snapshots of solutes around the colloid at $\epsilon_{\beta c}=0.8, \epsilon_{\beta c}=2.2$ and $\epsilon_{\beta c}=5.0$ shown in Figure 2.

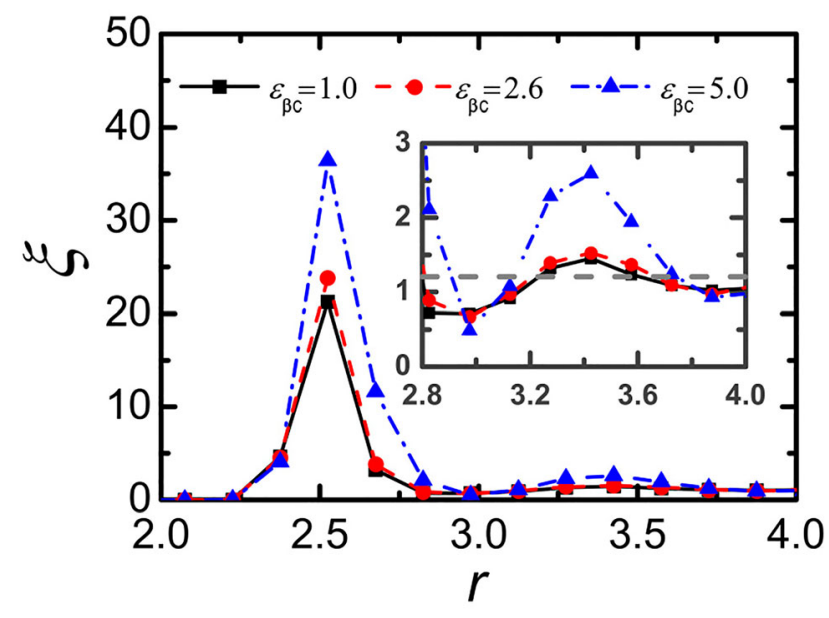

Figure 3. The auto-correlation for the shear terms of stress tensor, $\xi$, as a function of distance to the colloid, $r$, at three different $\epsilon_{\beta c}$. The inset magnifies $2.8<r<4.0$ part, with grey dashed line indicating the viscosity for system consists of pure $L J$ particles of $\sigma=1.0$ and $\epsilon=1.0$ at the same thermodynamic state.

To validate the existence of shell-region, Figure 3 presents the dependence of auto-correlation for the shear terms of stress tensor $\xi$ as a function of distance to the colloid $r$, which reflects the local viscosity of the fluid. While $\xi(r)$ is barely changed when $\epsilon_{\beta c}$ is increased from 1.0 to 2.6 , its peak around $r \sim 2.5$ mounts up when $\epsilon_{\beta c}$ is increased to 5.0. The peak is generated by the bound particles (mainly solvent), caged by the solute shell formed around $r \sim 3.0$, which is consistent with the position of the first peak of $F_{x}(r)$ in Figure S2 shown in SI. The magnified inset of Figure 3 presents the secondary peak around $r \sim 3.4$, at which the magnitude of $\xi$ for $\epsilon_{\beta c}=5.0$ is still larger than the other two cases. This peak is generated by the aggregation of solute particles adsorbed to the shell, constrained by the solute-colloid attraction, in accord with the peak of $F_{x}$ at the same position. For $r>4.0$, the value of $\xi(r)$ fluctuates around 1.21, i.e. the viscosity of pure LJ particles at same thermodynamic state obtained by the same method (Equation (4)), indicated by the grey dashed line within inset.

The results obtained here by simulation deviate from the continuum approximation $[29,47]$, for which the magnitude of $v_{x}$ is determined by

$$
\begin{aligned}
v_{x}= & \frac{2 \sigma_{c} T}{9 \eta} \int_{\sigma_{c}}^{\infty}\left[\left(\frac{\sigma_{c}}{2 r}\right)-\left(\frac{3 r}{2 \sigma_{c}}\right)\right. \\
& \left.+\left(\frac{r}{\sigma_{c}}\right)^{2}\right] A_{1}(r) \frac{\mathrm{d} U_{\beta}(r)}{\mathrm{d} r} \mathrm{~d} r
\end{aligned}
$$

where $\sigma_{c}$ is the radius of colloid, $\eta$ the viscosity, $A_{1}(r)$ the asymptotic expression for the first Legendre coefficient and $U_{\beta}(r)$ the solute-colloid interaction. Because 


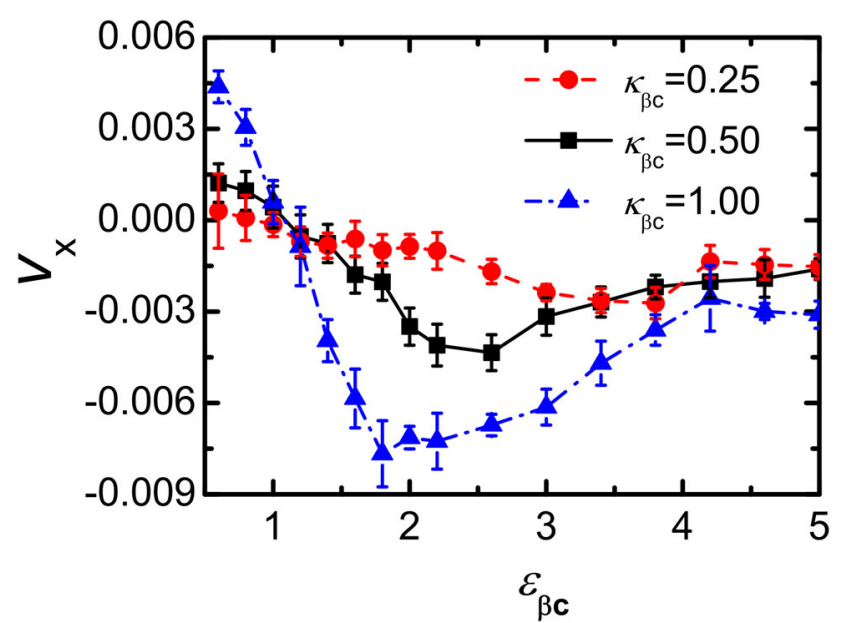

Figure 4. The dependence of the diffusio-phoretic flow velocity $v_{X}$ on $\epsilon_{\beta c}$, for different values of the range $\kappa_{\beta c}$ of the solute-colloid interaction.

the formation of shell-region is not taken into account, $v_{x}$ depends linearly on $\epsilon_{\beta c}$ according to Equation (5). See Figures S1-S4 of SI for more results and discussions on the effect of $\epsilon_{\beta c}$ on the diffusio-phoretic motion of colloid.

The magnitude of the phoretic flow also depends on the interaction range. Figure 4 shows the relation between $v_{x}$ and $\epsilon_{\beta c}$ for various values of $\kappa_{\beta c}$, the range parameter of the solute-colloid interaction. As is to be expected, the magnitude of $v_{x}$ is larger for larger $\kappa_{\beta c}$ (i.e. larger interactive width), as more solute particles would get attracted within the interface for larger $\kappa_{\beta c}$, a longer range of attraction results in a larger number of adsorbed solute particles. The maximum velocity $v_{x}^{m}$ is reached at smaller $\epsilon_{\beta c}$ for larger $\kappa_{\beta c}$.

The dependence of $v_{x}$ on solvent-colloid interactions is discussed in Figure S6 in SI.

\subsection{Density of solute}

Figure 5(a) shows the dependence of $v_{x}$ on $\epsilon_{\beta c}$, at different solute density in bulk, namely $\rho_{\beta}^{\text {bulk }}=0.05, \rho_{\beta}^{\text {bulk }}=$ 0.25 and $\rho_{\beta}^{b u l k}=0.50$. Correspondingly, the number fraction of solutes in bulk are $\phi_{\beta}^{\text {bulk }}=0.066, \phi_{\beta}^{b u l k}=$ 0.328 and $\phi_{\beta}^{b u l k}=0.655$. For $\epsilon_{\beta c}<2.0$ and $\epsilon_{\beta c}>4.0$, the value of $v_{x}\left(\epsilon_{\beta c}\right)$ barely depends on $\rho_{\beta}^{\text {bulk }}$. For $2.0<$ $\epsilon_{\beta c}<4.0$, the magnitude of $v_{x}$ is markedly larger for $\rho_{\beta}^{b u l k}=0.05$. When $\rho_{\beta}^{\text {bulk }}>0.25$, the flow velocity saturates at around $v_{x}=-0.002$ for $\epsilon_{\beta c}>2.0$. The weaker diffusio-phoresis for larger bulk density of solutes is due to two factors, both related to the fact that the diffusiophoretic velocity $v_{x}$ is equal to the product of the gradient of the chemical potential of the solute $\partial \mu_{\beta}^{b u l k} / \partial x$ and the excess density of solute in the adsorption layer around the

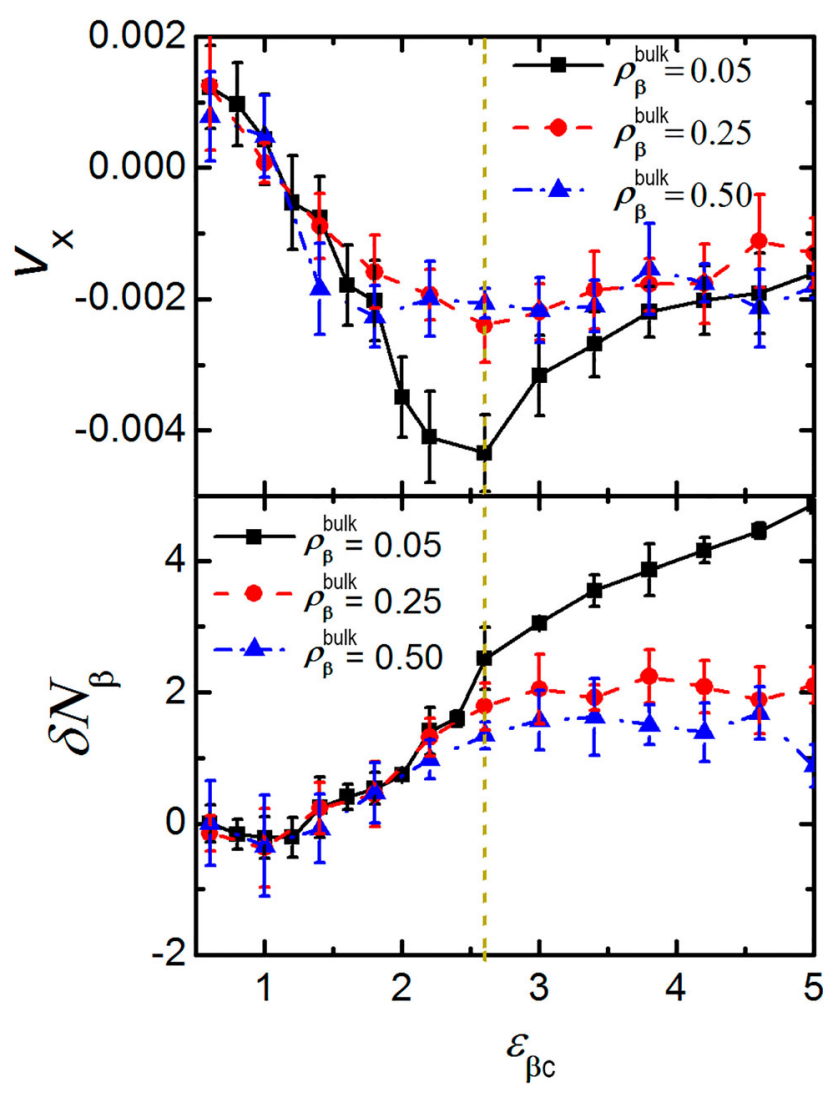

Figure 5. (a) The dependence of diffusio-phoretic flow velocity $v_{x}$, on the interaction strength between solute and colloid, $\epsilon_{\beta c}$, for different solute densities $\rho_{\beta}^{\text {bulk }}$. (b) Number difference of solute particles of left $(x<0)$ and right $(x>0)$ part of the system $\delta N_{\beta}$ as a function of $\epsilon_{\beta c}$ at different $\rho_{\beta}^{\text {bulk }}$. The dashed line indicates $\epsilon_{\beta c}=2.6$ at which the magnitude of $v_{x}$ reaches maximum.

colloid [50]

$$
v_{x}=\frac{1}{\eta} \frac{\partial \mu_{\beta}^{b u l k}}{\partial x} \int_{\sigma_{c}}^{\infty} r\left[\rho_{\beta}(r)-\rho_{\beta}^{b u l k}\right] \mathrm{d} r
$$

where $\rho_{\beta}(r)$ and $\rho_{\beta}^{b u l k}$, respectively, denote the solute density at distance $r$ to the fixed colloid and in bulk region. As solvent and solute form an ideal mixture, this gradient is simply $\nabla \rho_{\beta} / \rho_{\beta}^{b u l k}$, which at constant $\nabla \rho_{\beta}$ is inversely proportional to $\rho_{\beta}^{\text {bulk }}$. The second reason is that the excess of solute in the adsorption layer $\rho_{\beta}^{e x}=\rho_{\beta}^{\text {local }}$ $\rho_{\beta}^{b u l k}$ tends to saturate rather quickly with $\epsilon_{\beta c}$. Once that happens, increasing $\rho_{\beta}^{b u l k}$ tends to decrease $\rho_{\beta}^{\text {ex }}$ near the colloid. This effect is counteracted to some extent by the fact that for sufficiently large $\epsilon_{\beta c}$, the total number of adsorbed particles increases.

The increase of $\rho_{\beta}^{b u l k}$ also leads to the decrease of the asymmetric distribution of solutes around the colloid in the presence of the gradient forces. This is illustrated 


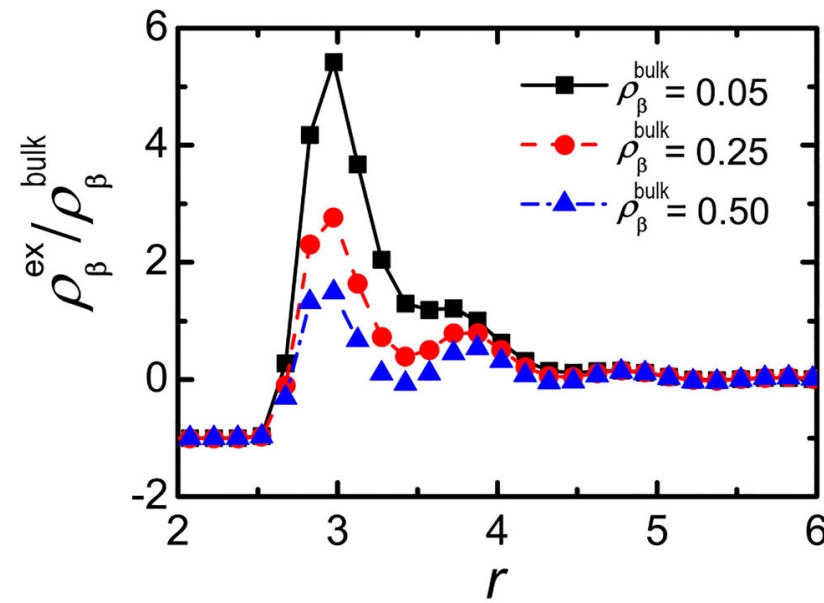

Figure 6. The normalised excess density of solute particles $\rho_{\beta}^{e x} / \rho_{\beta}^{b u l k}$ as a function of $r$ at $\epsilon_{\beta c}=2.6$.

in Figure 5(b). The number difference of solute particles at left $(x<0)$ and right $(x>0)$ part of the system $\delta N_{\beta}$ increases obviously with $\epsilon_{\beta c}$ for $\rho_{\beta}^{\text {bulk }}=0.05$. However, for $\rho_{\beta}^{\text {bulk }} \geq 0.25$, the value of $\delta N_{\beta}$ saturates near $\epsilon_{\beta c}=2.6$, which is also the point where the diffusiophoretic flow velocity appears to maximise (indicated by the dashed line). The dependence of asymmetry of solutes distribution on $r$ is also discussed in Figure S7 of SI.

Figure 6 shows the saturation at high values of $\epsilon_{\beta c}$ of the normalised excess density of solute particles adsorbed to the colloid. Upon increasing the solute density in

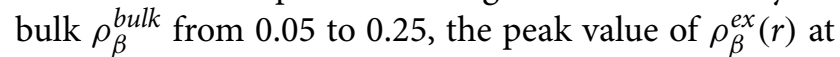
$r=3.0$ clearly decreases. However, upon further increase in $\rho_{\beta}^{b u l k}$, the excess density of the adsorbed solute is changed very slightly.

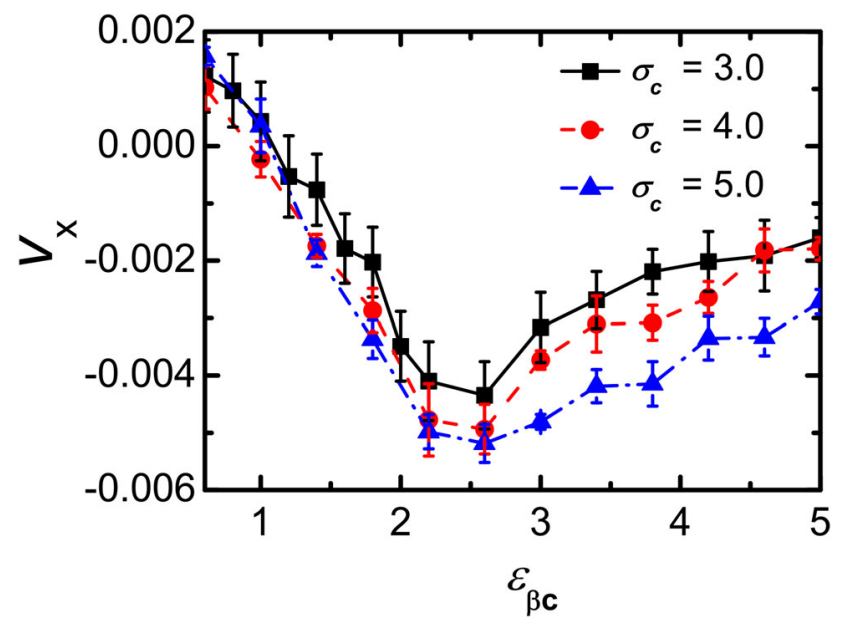

Figure 7. The dependence of velocity in $x$-direction of fluids, $v_{x}$, on the interaction strength between solute and colloid, $\epsilon_{\beta c}$, for different colloid sizes $\sigma_{c}$.

\subsection{Size effect}

The dependence of $v_{x}$ on the size of the colloid $\sigma_{c}$, controlled by setting the position of the minimum of the Morse potential as $\sigma_{\alpha c} \equiv \sigma_{\beta c} \equiv \sigma_{c}$, is shown in Figure 7 . Note that the width of the potential well is kept constant, with fixed variations in the truncated cut-off distance and the size of colloid as $\delta r^{t}=\delta \sigma$. Increasing the size of the colloid increases the magnitude of the diffusio-phoretic flow (for any given $\epsilon_{\beta c}$ ). Such an effect is to be expected, as the volume of the adsorption region increase with $\sigma_{\beta c}$ and the formation of shell-region depends mainly on $\epsilon_{\beta c}$.

\section{Conclusions}

To conclude, in this work, how the diffusio-phoretic motion of colloidal particle gets suppressed by the formation of the shell-region is explained numerically. Although the excess density of adsorbed solute increases with the strength of solute-colloid attraction, this effect is counteracted by the fact that very strong binding of the solute suppresses diffusio-phoresis, as the adsorbed layer becomes more viscous. The optimal solute-colloid attraction that maximises the phoretic mobility is thus determined. It is also found that the phoretic motion can be impeded at high solute density in bulk, as both the degree of asymmetric distribution of solutes surrounding the colloid and the excess density of solutes in the adsorption shell decrease with solute density in bulk. The phoretic mobility is also found to depend on interaction range, size and hydrophobicity of the colloid, all of which change the concentration distribution of solutes (or solvents) surrounding the colloid. Similarly, introducing another colloidal particle would also change the magnitude of flow velocity with a perturbation on the concentration field. See Figure S8 in SI for more discussions. The results indicate that diffusio-phoresis may be applied to the selective transport, separation and purification of biomacromolecules and other types of colloids, due to the sensitive dependence of the diffusio-phoretic mobility on the interaction strength and the density of solutes. However, as the magnitude of flow velocity increases with the solute-colloid interaction range, it might be more efficient to transport bio-macromolecules such as protein or DNA in a concentration gradient of electrolyte with long-range interactions, i.e. by eletro-phoresis.

\section{Acknowledgments}

The author appreciates illuminating comments from Prof. Daan Frenkel and fruitful discussions with Yawei Liu, Raman Ganti and Simon Ramirez.

\section{Disclosure statement}

No potential conflict of interest was reported by the author. 


\section{Funding}

This work was supported by National Natural Science Foundations of China [grant number 2016YFA0501601] and National Key Research and Development Program of China [grant number 11602279].

\section{References}

[1] J.C. Eijkel and A. van den Berg, Chem. Soc. Rev. 39, 957 (2010).

[2] J.L. Anderson and D.C. Prieve, Sep. Purif. Methods 13, 67 (2006).

[3] O. Annunziata, D. Buzatu and J.G. Albright, J. Phys. Chem. B 116, 12694 (2012).

[4] J. Deseigne, C. Cottin-Bizonne, A.D. Stroock, L. Bocquet and C. Ybert, Soft Matter 10, 4795 (2014).

[5] J. Palacci, B. Abecassis, C. Cottin-Bizonne, C. Ybert and L. Bocquet, Phys. Rev. Lett. 104, 138302 (2010).

[6] J.S. Paustian, C.D. Angulo, R. Nery-Azevedo, N. Shi, A.I. Abdel-Fattah and T.M. Squires, Langmuir 31, 4402 (2015).

[7] D.C. Prieve, S.M. Malone, A.S. Khair, R.F. Stout and M.Y. Kanj, Proc. Natl Acad. Sci. USA 116, 18257 (2019).

[8] M. Yang, A. Wysocki and M. Ripoll, Soft Matter 10, 6208 (2014).

[9] Y. Zhao, C. Zhao, J. He, Y. Zhou and C. Yang, Soft Matter 9, 7726 (2013).

[10] A. Wurger, Rep. Progr. Phys. 73, 126601 (2010).

[11] M. Braibanti, D. Vigolo and R. Piazza, Phys. Rev. Lett. 100, 108303 (2008).

[12] J. Burelbach, D. Frenkel, I. Pagonabarraga and E. Eiser, Eur. Phys. J. E 41, 7 (2017).

[13] J. Burelbach, M. Zupkauskas, R. Lamboll, Y. Lan and E. Eiser, J. Chem. Phys. 147, 094906 (2017).

[14] L. Fu, S. Merabia and L. Joly, Phys. Rev. Lett. 119, 214501 (2017).

[15] Y.T. Maeda, A. Buguin and A. Libchaber, Phys. Rev. Lett. 107, 038301 (2011).

[16] R. Piazza, Soft Matter 4, 1740 (2008).

[17] R. Piazza and A. Parola, J. Phys.: Condens. Matter 20, 153102 (2008).

[18] T. Tsuji, K. Kozai, H. Ishino and S. Kawano, Micro Nano Lett. 12, 520 (2017).

[19] D. Vigolo, R. Rusconi, H.A. Stone and R. Piazza, Soft Matter 6, 3489 (2010).

[20] D. Vigolo, J. Zhao, S. Handschin, X. Cao, A.J. deMello and R. Mezzenga, Nat. Sci. Rep. 7, 1211 (2017).

[21] C.J. Wienken, P. Baaske, U. Rothbauer, D. Braun and S. Duhr, Nat. Commun. 1, 100 (2010).

[22] M. Wolff, J.J. Mittag, T.W. Herling, E.D. Genst, C.M. Dobson, T.P. Knowles, D. Braun and A.K. Buell, Nat. Sci. Rep. 6, 22829 (2016).
[23] T.Y. Molotilin, V. Lobaskin and O.I. Vinogradova, J. Chem. Phys. 145, 244704 (2016).

[24] I. Semenov, S. Raafatnia, M. Sega, V. Lobaskin, C. Holm and F. Kremer, Phys. Rev. E 87, 022302 (2013).

[25] R.F. Stout and A.S. Khair, Phys. Rev. Fluids 2, 014201 (2017).

[26] M. Tanaka and A.Y. Grosberg, Eur. Phys. J. E 7, 371 (2002).

[27] M. Tanaka, Phys. Rev. E 68, 061501 (2003).

[28] K.L. Saar, Y. Zhang, T. Muller, C.P. Kumar, S. Devenish, A. Lynn, U. Lapinska, X. Yang, S. Linse and T.P.J. Knowles, Lab Chip 18, 162 (2017).

[29] J.L. Anderson, J. Colloid Interface Sci. 82, 248 (1981).

[30] A.S. Khair, J. Fluid. Mech. 731, 64 (2013).

[31] R.P. Sear and P.B. Warren, Phys. Rev. E 96, 062602 (2017).

[32] D. Velegol, A. Garg, R. Guha, A. Kar and M. Kumar, Soft Matter 12, 4686 (2016).

[33] B. Abecassis, C. Cottin-Bizonne, C. Ybert, A. Ajdari and L. Bocquet, Nat. Mater. 7, 785 (2008).

[34] J.T. Ault, P.B. Warren, S. Shin and H.A. Stone, Soft Matter 13, 9015 (2017).

[35] J.T. Ault, S. Shin and H.A. Stone, J. Fluid. Mech. 854, 420 (2018).

[36] S. Shin, J.T. Ault, P.B. Warren and H.A. Stone, Phys. Rev. X 7, 041038 (2017).

[37] S. Shin, E. Um, B. Sabass, J.T. Ault, M. Rahimi, P.B. Warren and H.A. Stone, Proc. Natl Acad. Sci. USA 113, 257 (2016).

[38] A. Banerjee, I. Williams, R.N. Azevedo, M.E. Helgeson and T.M. Squires, Proc. Natl Acad. Sci. USA 113, 8612 (2016).

[39] N. Shi, R. Nery-Azevedo, A.I. Abdel-Fattah and T.M. Squires, Phys. Rev. Lett. 117, 258001 (2016).

[40] R. Sear, Phys. Rev. Lett. 122, 128101 (2019).

[41] M. Yang, R. Liu, F. Ye and K. Chen, Soft Matter 13, 647 (2017).

[42] T. Chen, C. Xu and Z. Ren, J. Ind. Manag. Optim. 13, 1 (2017).

[43] M.-J. Huang, J. Schofield and R. Kapral, Soft Matter 12, 5581 (2016).

[44] Y. Liu, R. Ganti and D. Frenkel, J. Phys.: Condens. Matter 30, 205002 (2018).

[45] H. Yoshida, S. Marbach and L. Bocquet, J. Chem. Phys. 146, 194702 (2017).

[46] R. Ganti, Y. Liu and D. Frenkel, Phys. Rev. Lett. 119, 038002 (2017).

[47] N. Sharifi-Mood, J. Koplik and C. Maldarelli, Phys. Rev. Lett. 111, 184501 (2013).

[48] Y. Liu, R. Ganti, H.G. Burton, X. Zhang, W. Wang and D. Frenkel, Phys. Rev. Lett. 119, 224502 (2017).

[49] S. Plimpton, J. Comput. Phys. 117, 1 (1995).

[50] J.L. Anderson, M.E. Lowell and D.C. Prieve, J. Fluid Mech. 117, 107 (1982). 